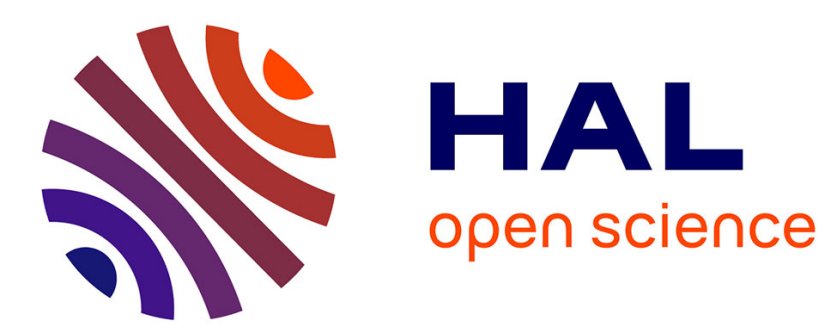

\title{
Characterization of a 3D defect using the Expected Improvement algorithm
}

S. Bilicz, Emmanuel Vazquez, Marc Lambert, S. Gyimothy, J. Pavo

\section{To cite this version:}

S. Bilicz, Emmanuel Vazquez, Marc Lambert, S. Gyimothy, J. Pavo. Characterization of a 3D defect using the Expected Improvement algorithm. 13th IGTE - Symposium on Numerical Field Calculation in Electrical Engineering, Sep 2008, Graz, Austria. hal-01104089v2

\section{HAL Id: hal-01104089 \\ https://hal-centralesupelec.archives-ouvertes.fr/hal-01104089v2}

Submitted on 19 Jan 2009

HAL is a multi-disciplinary open access archive for the deposit and dissemination of scientific research documents, whether they are published or not. The documents may come from teaching and research institutions in France or abroad, or from public or private research centers.
L'archive ouverte pluridisciplinaire HAL, est destinée au dépôt et à la diffusion de documents scientifiques de niveau recherche, publiés ou non, émanant des établissements d'enseignement et de recherche français ou étrangers, des laboratoires publics ou privés. 


\title{
Characterization of a 3D defect using the Expected Improvement algorithm
}

\author{
S. Bilicz ${ }^{*}$, E. Vazquez ${ }^{\dagger}$, M. Lambert $*$, S. Gyimothy ${ }^{\ddagger}$ and J. Pavo ${ }^{\ddagger}$ \\ * Département de Recherche en Électromagnétisme, Laboratoire des Signaux et Systèmes UMR8506 \\ (CNRS-SUPELEC-Univ Paris Sud 11), 91192 Gif-sur-Yvette cedex, France \\ $\dagger$ SUPELEC, 91192 Gif-sur-Yvette cedex, France \\ \$ Budapest University of Technology and Economics, Egry J. u. 18, H-1521 Budapest, Hungary \\ E-mail: biliczs@sch.bme.hu
}

\begin{abstract}
This paper provides a new methodology for the characterization of a defect embedded in a conductive nonmagnetic plate from the measurement of the impedance variations of an air-cored pancake coil at eddy current frequencies. The inversion problem is dealt with using the Expected Improvement (EI) global optimization algorithm. The efficiency of the approach is discussed in the light of preliminary numerical examples obtained using synthetic data.
\end{abstract}

Keywords: ENDE, global optimization, inverse problem, response surface, kriging

\section{INTRODUCTION}

This paper deals with the characterization of a 3D defect inside a flat isotropic non-magnetic metal plate, from measured variations of the impedance of an eddy current testing (ECT) probe coil driven by low-frequency timeharmonic current. The probe is an air-cored, pancake-type coil, moved above a damaged zone. The data consists of a map of the variations of the coil impedance due to the presence of the defect, at discrete locations of a planar surface above and parallel to the plate.

As illustrated by a large number of contributions in the last ten years, the retrieval of 3D scattering objects - such as defects - remains quite challenging from a theoretical, computational and/or experimental perspective, even in the case of the simplest configurations (e.g., a homogeneous embedding space). In this paper, an optimization-based inversion method is presented. We use the so-called Expected Improvement (EI) algorithm to perform the optimization task. The EI algorithm is well-known in the domain of global optimization, but to the best of our knowledge, it is the first time that it is used for the ECT problem.

First, we present the forward problem and its numerical simulation. Then the inverse problem - along with our regularizing assumptions - will be presented. Next the new stochastic approach and the optimization task will be in the focus. Finally, numerical examples will illustrate the method proposed and the conclusions of the experiments will be drawn.

\section{The FORWARD PROBLEM}

The object to be analyzed is a homogeneous, nonmagnetic infinite metal plate with conductivity $\sigma_{0}$ and thickness $d$. We assume the presence of a volumetric material flaw inside the plate in a region denoted by $V^{d}$, which changes the electric conductivity locally. An electric field is generated by the ECT probe coil, which is an air-cored, pancake-type coil with turns parallel to the plate. The impedance of the coil changes due to the presence of the flaw. The impedance variation is the output signal. The forward problem is the determination of the output signal knowing all the characteristics of the scanned object.

\section{A. Mathematical model}

The solution of the forward problem is obtained by the classical volume integral approach [1] and a field decomposition. The electric field at a position $\mathbf{r}$ in the plate is written as a sum of two terms:

$$
\mathbf{E}(\mathbf{r})=\mathbf{E}^{i}(\mathbf{r})+\mathbf{E}^{d}(\mathbf{r}),
$$

where $\mathbf{E}^{i}$ is the incident field (in a flawless plate), and $\mathbf{E}^{d}$ is the defect field, i.e. the distortion of the field due to the flaw. The current dipole densities $\mathbf{P}$ and $\mathbf{P}^{\mathbf{i}}$ are defined by

$$
\mathbf{P}(\mathbf{r})=\left(\sigma(\mathbf{r})-\sigma_{0}\right) \mathbf{E}(\mathbf{r}) \text { and } \mathbf{P}^{i}(\mathbf{r})=\left(\sigma(\mathbf{r})-\sigma_{0}\right) \mathbf{E}^{i}(\mathbf{r}) .
$$

The interaction of the EM field of the coil with the flaw can be described by an integral equation that operates a coupling of the two terms in (1) on the domain $V^{d}$ :

$$
\mathbf{P}(\mathbf{r})=\mathbf{P}^{i}(\mathbf{r})-j \omega \mu_{0} \sigma_{0} \chi(\mathbf{r}) \int_{V^{d}} \mathcal{G}\left(\mathbf{r} \mid \mathbf{r}^{\prime}\right) \mathbf{P}\left(\mathbf{r}^{\prime}\right) \mathrm{d} V^{\prime} .
$$

The so-called defect description function $\chi(\mathbf{r})$ is defined as

$$
\chi(\mathbf{r})=\frac{\sigma(\mathbf{r})-\sigma_{0}}{\sigma_{0}} .
$$

The notation $\mathcal{G}\left(\mathbf{r} \mid \mathbf{r}^{\prime}\right)$ refers to the related dyadic Green's function which transforms the current dipole excitation at $\mathbf{r}^{\prime}$ into the generated electric field at $\mathbf{r}$. Once (2) is solved, the variation of the coil impedance can be expressed as

$$
\Delta Z=-\frac{1}{I_{0}^{2}} \int_{V^{d}} \mathbf{E}^{i}(\mathbf{r}) \cdot \mathbf{P}(\mathbf{r}) \mathrm{d} V
$$

based on the reciprocity theorem. Here, $I_{0}$ denotes the current of the probe coil.

\section{B. Numerical simulation}

A MATLAB code has been developed to solve the forward problem. The integral equation (2) is solved by a Galerkin version of a Method of Moments using pulse basis functions (the defect is discretized using a set of uniform, cuboid-shaped disjunct cells and the unknown $\mathbf{P}$ current dipole density is assumed to be constant in each of them). In so doing the integral equation (2) is transformed into a system of linear equations, which can be written in a matrix form and where the unknowns are the approximate values of $\mathbf{P}$ in each cell, the entries of the left-hand side matrix are approximations of $\mathcal{G}\left(\mathbf{r} \mid \mathbf{r}^{\prime}\right)$, and the entries 
of the right-hand side are the excitations of the system related to the incident field. The latter can be expressed in analytical form in the case of the simple configuration we have chosen. $\mathcal{G}\left(\mathbf{r} \mid \mathbf{r}^{\prime}\right)$ is expressed and integrated in closedform in the spectral domain, and is calculated in the spatial domain via FFT.

A considerable advantage of the field decomposition method is that it separates the computation of the incident field and of the Green's function. The latter is a relatively time-consuming task, but it has to be performed only once - then the system matrix related to any defect can be easily assembled from the stored data (obviously, for the same plate parameters, frequency and cell sizes). Beyond the fast generation of the equation system for an arbitrary defect, the computation of the variation of coil impedance at a lagre number of locations does not take practically more time than the computation at only one single location since the system matrix remains the same when the coil is moving from point to point.

\section{The INVERSE PROBLEM}

When the probe coil scans the zone containing the flaw, the variations of the coil impedance are measured at each point of a regular rectangular grid. The problem is to characterize the flaw from the set of measured impedance variations, which will now be viewed as the input data of the inverse problem.

\section{A. Regularizing assumptions}

It is classical to introduce some regularizing assumptions to reduce the ill-posedness of the inverse problem. Here, we suppose a cuboid-shaped homogeneous material flaw, with known orientation - the defect has sides parallel to the plate surfaces. A defect is then characterized by seven parameters depicted by Fig. 1: the position of the flaw is described by three parameters $\left(x_{c}\right.$ and $y_{c}$ denote the center point on the $x-y$ plane, $z_{t}$ is the position of the upper side), the edge lengths are described by three parameters ( $L_{x}, L_{y}$ and $L_{z}$ in the related direction) and one parameter describes the conductivity $(\sigma)$. In the examples presented in this paper, $\sigma$ is assumed to be zero (air void), which reduces the number of parameters to be estimated to only six geometrical parameters. We will see that there is no practical difficulty in treating seven or even more unknown defect parameters. To simplify the notations, the unknown defect parameters are collected in a vector $\mathbf{p}$ :

$$
\mathbf{p}=\left[x_{c}, L_{x}, y_{c}, L_{y}, z_{t}, L_{z}\right] .
$$

The definition domain of $\mathbf{p}$ will be denoted by $\mathbb{P}$, and will be referred to as the parameter space.

A surface scan consists in moving the coil at $N$ different locations above a region of interest (ROI) (see Fig. 1). The (simulated) variation of the coil impedance at location $k$ induced by a cuboid-shaped defect characterized by its parameter vector $\mathbf{p}$ will be denoted by $\Delta Z_{k}(\mathbf{p})$, whereas the measured impedance variation at the same location will be denoted by $\Delta \mathcal{Z}_{k}$.

\section{B. Optimization task}

The next step for solving the inverse problem is to achieve the strongest resemblance between the impedance

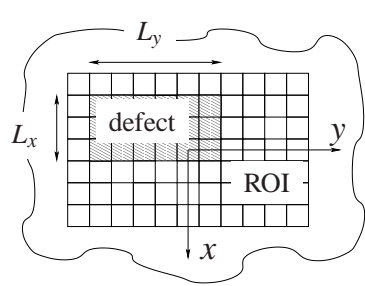

(a) On the $x-y$ plane.

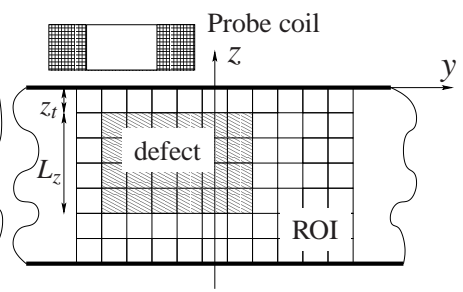

(b) Along the $z$ axis.
Figure 1. The ROI in the plate contains the assumed defect which has six geometric parameters.

signal obtained by simulation, $\left\{\Delta Z_{k}(\mathbf{p}), k=1, \ldots, N\right\}$, and the measured one, $\left\{\Delta \mathcal{Z}_{k}, k=1, \ldots, N\right\}$, by tuning the parameter vector $\mathbf{p}$.

The parameter space $\mathbb{P}$ consists of $M$ discrete parameter points. For instance, a 2-dimensional section of $\mathbb{P}$ in the $y_{c}-L_{y}$ plane is represented in Fig. 2 .

To give a mathematical form to the resemblance between two surface-scan impedance signals, we define the similarity function

$$
Q(\mathbf{p})=\sum_{k=1}^{N}\left|\Delta Z_{k}(\mathbf{p})-\Delta \boldsymbol{Z}_{k}\right|^{2} / \sum_{k=1}^{N}\left|\Delta \boldsymbol{Z}_{k}\right|^{2} .
$$

Our objective is thus to minimize $Q(\mathbf{p})$, i.e. to find

$$
\hat{\mathbf{p}}=\arg \min _{\mathbf{p} \in \mathbb{P}} Q(\mathbf{p}) \text {. }
$$

\section{Stochastic Modeling AND OPTimization OF The SIMILARITY FUNCTION}

Our objective is to implement the Expected Improvement algorithm to minimize $Q(\mathbf{p})$. One iteration of such an algorithm involves mainly two steps:

1) the construction of an approximation of the similarity function from a set of past evaluations of the function obtained at previous iterations. To this end, a random process is chosen as a model of the similarity function and an interpolation by kriging is performed.

2) the search of the maximum of the Expected Improvement over the parameter space $\mathbb{P}$.

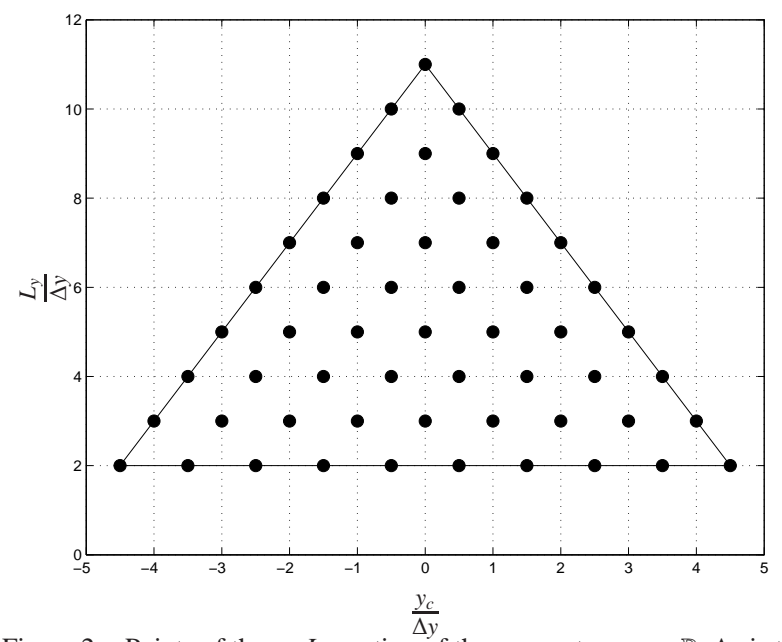

Figure 2. Points of the $y_{c}-L_{y}$ section of the parameter space $\mathbb{P} . \Delta y$ is the cell size along $y$ of the ROI discretization. The figure is related to Fig. 1 - the number of ROI cells along $y$ is 11 and defects smaller than $2 \Delta y$ are ignored. 
This two-step procedure is repeated iteratively until a stopping criterion is met, i.e. a sequential optimization algorithm is obtained.

\section{A. Kriging interpolation}

Let us assume that we have already observed the multivariate scalar function $Q(\mathbf{p})$ at $n$ points $\mathbf{p}_{1}, \mathbf{p}_{2}, \ldots, \mathbf{p}_{n}$ of $\mathbb{P}$. Then, $n$ function values $Q_{1}=Q\left(\mathbf{p}_{1}\right), Q_{2}=$ $Q\left(\mathbf{p}_{2}\right), \ldots, Q_{n}=Q\left(\mathbf{p}_{n}\right)$ are known. We would like to predict the function value at unobserved sites. One method to achieve this goal is to use kriging, a random process approach developed in the 60s in geostatistics [2]. The method is also well-known for modeling computer simulations [3].

Let $\xi(\mathbf{p})$ be a Gaussian random process that models the function $Q(\mathbf{p})$. Thus, each observation $Q_{k}$ is considered as the realization of the Gaussian random variable $\xi\left(\mathbf{p}_{k}\right)(k=$ $1,2, \ldots, n)$. Kriging computes the best linear unbiased predictor $(B L U P)$ of $\xi(\mathbf{p})$. Let us denote this prediction by $\hat{\xi}(\mathbf{p})$. The predictor is linear in the sense that it is a linear combination of the observed random variables $\xi\left(\mathbf{p}_{k}\right)$, $k=1,2, \ldots, n$, which can therefore be written as

$$
\hat{\xi}(\mathbf{p})=\sum_{k=1}^{n} \lambda_{k}(\mathbf{p}) \xi\left(\mathbf{p}_{k}\right) .
$$

Unbiasedness relates to the fact that the mean of $\hat{\xi}(\mathbf{p})$ is equal to the mean of $\xi(\mathbf{p})$, i.e. the mean prediction error is zero:

$$
\mathbb{E}[\varepsilon(\mathbf{p})]=\mathbb{E}[\hat{\xi}(\mathbf{p})-\xi(\mathbf{p})]=0
$$

The term "best" means that the prediction error $\varepsilon(\mathbf{p})$ of the kriging predictor has the smallest variance among all unbiased predictors. This variance (also called kriging error) may be written as

$$
\hat{\sigma}^{2}(\mathbf{p})=\operatorname{var}[\hat{\xi}(\mathbf{p})-\xi(\mathbf{p})]=\mathbb{E}\left[(\hat{\xi}(\mathbf{p})-\xi(\mathbf{p}))^{2}\right],
$$

using the unbiasedness condition (5).

The objective is to find the coefficients $\lambda_{k}(\mathbf{p})$ in (4) that achieve the BLUP. The kriging error can be written using the covariance function, which describes the dependence between two random variables of the process at different points. Let us denote the covariance function by $k\left(\mathbf{p}_{a}, \mathbf{p}_{b}\right)=\operatorname{cov}\left[\xi\left(\mathbf{p}_{a}\right), \xi\left(\mathbf{p}_{b}\right)\right]$, where $\mathbf{p}_{a}$ and $\mathbf{p}_{b}$ are two points in $\mathbb{P}$. Let us denote by $\mathbf{K}$, the covariance matrix whose entries correspond to the covariances of the random process between the observation points $\mathbf{p}_{1}, \mathbf{p}_{2}, \ldots, \mathbf{p}_{n}$ :

$$
\mathbf{K}=\left[\begin{array}{cccc}
k\left(\mathbf{p}_{1}, \mathbf{p}_{1}\right) & k\left(\mathbf{p}_{1}, \mathbf{p}_{2}\right) & \ldots & k\left(\mathbf{p}_{1}, \mathbf{p}_{n}\right) \\
k\left(\mathbf{p}_{2}, \mathbf{p}_{1}\right) & k\left(\mathbf{p}_{2}, \mathbf{p}_{2}\right) & \ldots & k\left(\mathbf{p}_{2}, \mathbf{p}_{n}\right) \\
\vdots & \vdots & \ddots & \vdots \\
k\left(\mathbf{p}_{n}, \mathbf{p}_{1}\right) & k\left(\mathbf{p}_{n}, \mathbf{p}_{2}\right) & \ldots & k\left(\mathbf{p}_{n}, \mathbf{p}_{n}\right)
\end{array}\right]
$$

If one has some prior knowledge on the function to be modeled it can be reflected by giving a prior mean to the predictor. Since in our case no information is available but the observed function values, a constant (but unknown) mean $\mathbb{E}[\xi(\mathbf{p})]=C$ is assumed.

To simplify the notations let us collect the coefficients $\lambda_{k}(\mathbf{p})$ into a vector $\lambda(\mathbf{p})=\left[\begin{array}{llll}\lambda_{1}(\mathbf{p}) & \lambda_{2}(\mathbf{p}) \ldots & \ldots & \lambda_{n}(\mathbf{p})\end{array}\right]^{\top}$, and denote by $\mathbf{k}(\mathbf{p})$ the vector whose elements are the values of the covariance between $\mathbf{p}$ and the observation points: $\mathbf{k}(\mathbf{p})=\left[k\left(\mathbf{p}, \mathbf{p}_{1}\right) k\left(\mathbf{p}, \mathbf{p}_{2}\right) \ldots k\left(\mathbf{p}, \mathbf{p}_{n}\right)\right]^{\top}$. It can be shown that the determination of $\boldsymbol{\lambda}(\mathbf{p})$ boils down to computing the solution of the linear system of equations (see, e.g., [4]):

$$
\left[\begin{array}{ccc|c} 
& & & 1 \\
& \mathbf{K} & & \vdots \\
& & & 1 \\
\hline 1 & \ldots & 1 & 0
\end{array}\right]\left[\begin{array}{l}
\lambda(\mathbf{p}) \\
\hline \mu(\mathbf{p})
\end{array}\right]=\left[\begin{array}{c}
\mathbf{k}(\mathbf{p}) \\
\hline 1
\end{array}\right]
$$

where $\mu(\mathbf{p})$ is the Lagrange multiplier, which correspond to the enforcement of the unbiasedness condition.

Once the vector $\boldsymbol{\lambda}(\mathbf{p})$ has been computed, a predicted value of the similarity function $Q(\mathbf{p})$ can be written as

$$
\hat{Q}(\mathbf{p})=\sum_{k=1}^{n} \lambda_{k}(\mathbf{p}) Q_{k} .
$$

It is easy to show that the function $\mathbf{p} \mapsto \hat{Q}(\mathbf{p})$ interpolates $Q(\mathbf{p})$ at observed points. An interesting property of kriging, is that an estimate of the uncertainty of the prediction is available via the kriging error, which can be written as

$$
\hat{\sigma}^{2}(\mathbf{p})=k(\mathbf{p}, \mathbf{p})-\lambda(\mathbf{p})^{\top} \mathbf{k}(\mathbf{p})-\mu(\mathbf{p}) .
$$

This feature will be essential in the adaptive sampling strategy of the Expected Improvement algorithm.

\section{B. Covariance model}

Before focusing on the optimization algorithm, we mention very briefly how the covariance function is chosen in practice (see also [4]).

First, a simplifying assumption is made - as usually done in geostatistics [2] - namely that the random process is stationary. Then, the covariance function is a one-variate function $k(h)$ where $h$ is a distance between two points $\mathbf{p}_{a}, \mathbf{p}_{b} \in \mathbb{P}$. This distance needs not to be the classical Euclidean distance. In our case, the components of $\mathbf{p} \in \mathbb{P}$ are of different kinds. Thus, it is reasonable to use some anisotropic distance, which may be written as

$$
h=\sqrt{\sum_{d=1}^{D}\left(\frac{P_{a, d}-P_{b, d}}{\rho_{d}}\right)^{2}} .
$$

where $P_{a, d}$ and $P_{b, d}$ are the $d^{\text {th }}$ components of the vectors $\mathbf{p}_{a}$ and $\mathbf{p}_{b}$, respectively, and $D$ the dimension of $\mathbb{P}$. The parameters $\rho_{d}, d=1,2, \ldots, D$, represent the range of the covariance, or the typical correlation distance, in the direction of the $d^{\text {th }}$ component.

Second, a parameterized covariance function is chosen and its parameters are estimated using the data with a maximum likelihood method (see, e.g, [4]). We use the Matérn covariance function, which can be written as

$$
k(h)=\frac{\sigma^{2}}{2^{v-1} \Gamma(v)}(2 \sqrt{v} h)^{v} \mathcal{K}_{v}(2 \sqrt{v} h),
$$

where $\mathcal{K}_{v}$ is the modified Bessel function of the second kind of order $v$. The parameter $v$ controls the regularity of the random process - the higher the $v$, the more regular the process is. The parameter $\sigma^{2}$ is the variance of the process $\left(k(0)=\sigma^{2}\right)$. The parameters $\rho_{d}$ in (11) are also estimated by maximum likelihood (the covariance function has indeed $D+2$ parameters). 


\section{Expected Improvement}

Since the similarity function $Q$ (to be minimized) requires to compute the solution of a forward problem, the computational cost of evaluating $Q$ is non-negligible. Moreover, for an inversion method to be useful in practice, the computational burden of the method must not be too high. Thus, we wish to limit the number of evaluations of $Q$ in the inversion procedure, which means that the optimization method used to minimize $Q$ must be efficient.

The expected improvement (EI) algorithm is an iterative method to find the global minimizers of an expensive-toevaluate function [5]. The method is based on interpolation by kriging of the function to be optimized. Let us assume that $Q$ has been evaluated at $n$ points $Q_{1}=Q\left(\mathbf{p}_{1}\right), Q_{2}=$ $Q\left(\mathbf{p}_{2}\right), \ldots, Q_{n}=Q\left(\mathbf{p}_{n}\right)$. An iteration of the EI algorithm provides the location of the next evaluation.

First, an interpolation $\hat{Q}$ of $Q$ is computed by kriging from the set of past evaluations, along with the variance of the kriging error $\hat{\sigma}^{2}(\mathbf{p})$. Denote the current minimal value by $Q_{\min }=\min _{k=1, \ldots, n} Q_{k}$. Define the improvement over $Q_{\min }$ at a point $\mathbf{p} \in \mathbb{P}$ by

$$
I(\mathbf{p})=\max \left(0, Q_{\min }-Q(\mathbf{p})\right) .
$$

However, $Q(\mathbf{p})$ is unknown except at the $n$ evaluation points. Since $Q(\mathbf{p})$ is modeled by the Gaussian random process $\xi$, a natural idea is to express the expected value of $I(\mathbf{p})$, which is called the expected improvement and appears to have a very convenient analytical form:

$$
E I(\mathbf{p}) \equiv \mathbb{E}[I(\mathbf{p})]=\hat{\sigma}(\mathbf{p})[u \Phi(u)+\varphi(u)],
$$

where $\Phi(\cdot)$ is the normal cumulative distribution function, $\varphi(\cdot)$ is the normal density function, and $u$ is defined by

$$
u=\frac{Q_{\min }-\hat{Q}(\mathbf{p})}{\hat{\sigma}(\mathbf{p})} .
$$

The next evaluation point is chosen according to the highest value of the expected improvement. Since (13) is straightforward to compute, the maximization of the EI over $\mathbb{P}$ is not a problem in practice. A natural stopping criterion is when the $E I$ is smaller than a small positive number. Fig. 3 presents a kriging interpolation and corresponding EI criterion.

Note that the $E I$ algorithm is known to be consistent, i.e. the algorithm converges to the location of the global minimizer (under some assumptions) [6], [7]. In theory, the convergence rate is unknown but it appears in practice that this type of algorithm converges very rapidly, as illustrated in our numerical studies (see next section).

\section{Numerical EXAMPLES}

In this section a couple of numerical examples have been chosen to illustrate the presented inversion method. The parameters of the ECT configuration are shown in Table I. The surface scan consists of 3481 measured impedance values - observed at the nodes of a rectangular grid characterized by the parameters given in Table I. The center of the surface scan is at the origin of the $x-y$ coordinate system and the edges of the grid are parallel to the related coordinate axes.
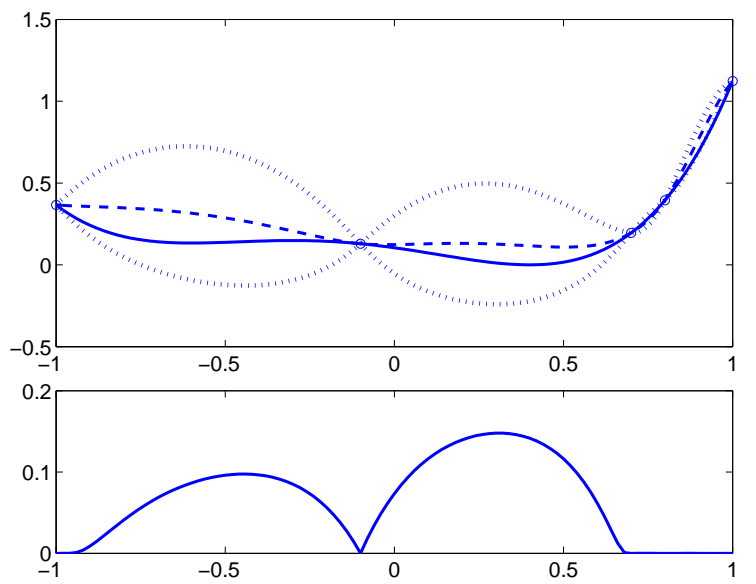

Figure 3. Illustration of a kriging interpolation and corresponding EI. Top: similarity function of one variable (continuous line), observations (circles), kriging prediction (dashed line), uncertainty - related to the predicted kriging error (dotted line). Bottom: the expected improvement. Note that the maximizer of the EI and the minimizer of the interpolation are not necessarily the same.

TABle I: Parameters of the ECT configuration.

\begin{tabular}{|l|l|l|l|}
\hline \multicolumn{4}{|c|}{ Metal plate } \\
\hline Thickness & $1.25 \mathrm{~mm}$ & Conductivity & $10^{6} \mathrm{~S} / \mathrm{m}$ \\
\hline \multicolumn{4}{|c|}{ Probe coil } \\
\hline Inner radius & $0.6 \mathrm{~mm}$ & Outer radius & $1.6 \mathrm{~mm}$ \\
Height & $0.8 \mathrm{~mm}$ & Lift-off & $0.5 \mathrm{~mm}$ \\
No. of turns & 140 & Frequency & $150 \mathrm{kHz}$ \\
\hline \multicolumn{4}{|c|}{ Surface scan } \\
\hline Points in the $x$ dir. & 59 & Points in the ydir. & 59 \\
Step in the $x$ dir. & $0.2 \mathrm{~mm}$ & Step in the y dir. & $0.2 \mathrm{~mm}$ \\
\hline
\end{tabular}

The ROI of all the performed experiments is the same: the number of cells along both the $x$ and $y$ directions is 7 , along the $z$ axis is 10 , respectively. Therefore, the total number of cells is 490. The center of the ROI on the $x-y$ plane is at the origin of the $x-y$ coordinate system as it is shown in Fig. 1. The cell sizes are: $\Delta x=\Delta y=0.2 \mathrm{~mm}$, $\Delta z=0.125 \mathrm{~mm}-$ i.e. the ROI takes place along the total thickness of the plate and has an extension of $1.4 \mathrm{~mm}$ along the $x$ and $y$ directions.

The number of cells of the ROI partly determines the 6dimensional parameter space on which the optimization is performed. The maximal defect sizes cannot exceed the size of the ROI, obviously. For physical - and plausible reasons, minimal defect sizes also have to be fixed. In our case, the minimal defect edge lengths are chosen as the double cell edge lengths along each direction. In this way, after some computation (notice the regularity in Fig. 2), one can see that the number of points of the discrete parameter space is 158,760 .

Since no real measurement was performed, the so-called "measured impedance variation" $\left(\Delta \mathcal{Z}_{k}, k=1, \ldots, N\right.$ in (3)) of the defects in the assumed test cases are obtained by numerical simulation. To avoid the inverse crime phenomenon the latter are computed using the CIVA simulation software [8] whereas our algorithm was used to compute the "simulated impedance variation" $\left(\Delta Z_{k}(\mathbf{p}), k=\right.$ $1, \ldots, N$ in (3)). In addition, the volume discretization used in CIVA is different (finer) from the cell sizes of our ROI.

We have seen that the EI algorithm needs an initialization at the beginning, i.e. the similarity function has to be 
TABLE II: THE INITIAL OBSERVATION POINTS.

\begin{tabular}{|c|c|c|c|c||c|c|c|}
\hline \multicolumn{7}{|c||}{$x-y$ plane } & \multicolumn{3}{c|}{$z$ axis } \\
\hline No. & $\frac{x_{c}}{\Delta x}$ & $\frac{L_{x}}{\Delta x}$ & $\frac{y_{c}}{\Delta y}$ & $\frac{L_{y}}{\Delta y}$ & No. & $\frac{z_{t}}{\Delta z}$ & $\frac{L_{z}}{\Delta z}$ \\
\hline \hline$\# 1$ & -2.5 & 2 & -2.5 & 2 & $\# 1$ & 0 & 5 \\
$\# 2$ & -2.5 & 2 & 2.5 & 2 & $\# 2$ & 3 & 5 \\
$\# 3$ & 2.5 & 2 & -2.5 & 2 & $\# 3$ & 5 & 5 \\
$\# 4$ & 2.5 & 2 & 2.5 & 2 & & & \\
$\# 5$ & 0 & 3 & 0 & 7 & & & \\
$\# 6$ & 0 & 7 & 0 & 3 & & & \\
$\# 7$ & 0 & 3 & 0 & 3 & & & \\
$\# 8$ & -2.5 & 2 & 0 & 7 & & & \\
$\# 9$ & 2.5 & 2 & 0 & 7 & & & \\
$\# 10$ & 0 & 7 & 2.5 & 2 & & & \\
$\# 11$ & 0 & 7 & -2.5 & 2 & & & \\
\hline
\end{tabular}

Table III: Parameters of the Defects to BE Characterized (0.1 MM, EXACT/RECONSTRUCTED).

\begin{tabular}{|c|c|c|c|c|c|c|r|}
\hline No. & $x_{c}$ & $L_{x}$ & $y_{c}$ & $L_{y}$ & $z_{t}$ & $L_{z}$ & It. \\
\hline \hline$\# 1$ & $2 / \mathbf{3}$ & $3 / \mathbf{4}$ & $-1 / \mathbf{0}$ & $7 / \mathbf{6}$ & $0 / \mathbf{0}$ & $2.5 / \mathbf{2 . 5}$ & 88 \\
\hline$\# 2$ & $2 / \mathbf{2}$ & $5 / \mathbf{6}$ & $-1 /-\mathbf{1}$ & $9 / \mathbf{8}$ & $0 / \mathbf{0}$ & $5 / \mathbf{5}$ & 69 \\
\hline$\# 3$ & $2 / \mathbf{1}$ & $5 / \mathbf{4}$ & $-1 /-\mathbf{2}$ & $9 / \mathbf{1 0}$ & $7.5 / \mathbf{6 . 2 5}$ & $5 / \mathbf{5}$ & 113 \\
\hline$\# 4$ & $0 / \mathbf{0}$ & $11 / \mathbf{1 4}$ & $0 / \mathbf{0}$ & $11 / \mathbf{1 4}$ & $5 / \mathbf{5}$ & $7.5 / \mathbf{5}$ & 31 \\
\hline$\# 5$ & $2 / \mathbf{2}$ & $5 / \mathbf{6}$ & $-1 / \mathbf{0}$ & $9 / \mathbf{6}$ & $3.75 / \mathbf{3 . 7 5}$ & $5 / \mathbf{5}$ & 81 \\
\hline
\end{tabular}

observed at some points before starting the iterative loop. There is no "best" choice of these initial points. Obviously, it is reasonable to spread them more or less uniformly on the parameter space. Too few points can give misleading information on the similarity function whereas too many points may be unnecessary (thus "uneconomical" in the sense of computation time). In all of our experiments here, 33 initial points have been chosen. They are summarized in Table II: 11 points on the 4D $x_{c}-L_{x}-y_{c}-L_{y}$ subspace and 3 points on the $2 \mathrm{D} z_{t}-L_{z}$ subspace. All the possible pairs of points from these two sets are taken therefore totally we have 33 points.

The algorithm has been applied on five different defects, two inner cracks (\#1-\#2), two outer cracks (\#3-\#4) and a buried one. Their physical parameters are summarized in Table III - along with the values retrieved by the inversion algorithm. In the last column, the number of the iteration cycle, in which the minimum similarity function value was found, is presented. Note that the iteration starts after the 33 initial function evaluation. One can see in Table III that the inversion algorithm performs well in the sense that

1) the retrieved defect parameters are quite close to the real ones. (Note that in some cases exact matching is not possible due to the discretization of the ROI and so the parameter space.)

2) the number of function evaluations needed to get the minimizer is considerably low in comparison with the total number of points of the parameter space $(158,760)$.

Obviously, 1) proves that the applied forward solver is precise, that the inversion has been traced back correctly to an optimization task and that the EI algorithm finds the global minimizer of the similarity function. Moreover, 2) allows us to think that the EI algorithm is quite effective.

The performance of the algorithm is illustrated in Fig. 4 and Fig. 5. On the bottom diagrams, the maximal expected improvement (i.e. over the total discrete parameter space) at the related iteration cycle is presented. One can see that it is decreasing as the iterations are being performed. However, the decrease is not monotonous. The difficulty of setting a correct stopping limit to the EI is obvious: in the case of defect No. \#4 an EI limit of $10^{-3}$ would work well, but the same limit in the case of defect No. \#1 would make the algorithm miss the solution.

One may notice a strange phenomenon in Fig. 4. At the iteration cycle No. 44, a very small similarity function value is obtained - approximately the same value as the final solution at cycle No. 88. The assumed defects corresponding to these cycles are very close to each other and to the real defect, obviously. However, in the EI algorithm, the pure value of the objective function is involved directly neither in the choice of the next point nor in the stopping criterion - as it has been expounded.

When the iterations are completed, the parameter point corresponding to the minimal observed similarity function value (which is also marked in the figures) is returned as the solution of the inverse problem.

The computations were performed on a PC with $16 \mathrm{~Gb}$ RAM and a 64 bits CPU at $3 \mathrm{GHz}$. The computation of the Green's function and of the incident field was made in advance, separately. One average iteration cycle of the optimization loop took approximately $70 \mathrm{sec}$, of which $20 \%$ is the evaluation of the similarity function and $80 \%$ is for the stochastic tools.
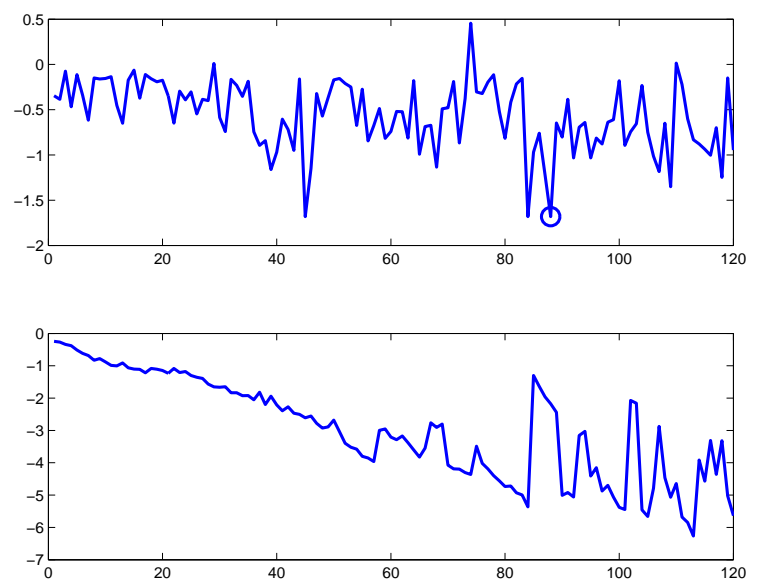

Figure 4. Defect No. \#1. Top: log of the current similarity function value. Minimizer is marked. Bottom: log of the maximal expected improvement. Both diagrams are in the function of the iteration number.

\section{Conclusions}

A new methodology in ECT inversion was presented. The stochastic-based optimization algorithm was coupled with a volume integral-based forward solver - the developed inversion method seems to be efficient in the light of the presented test cases.

The use of the kriging interpolation can be regarded as an alternative way of electromagnetic field computation. In fact, the kriging interpolation of the similarity function (which is the output of an electromagnetic "black-box") makes possible to have a view of the similarity function at unobserved points, i.e. without classical field computation. In other words, the forward simulator (complicated, time-consuming to run) is replaced in some sense with an 

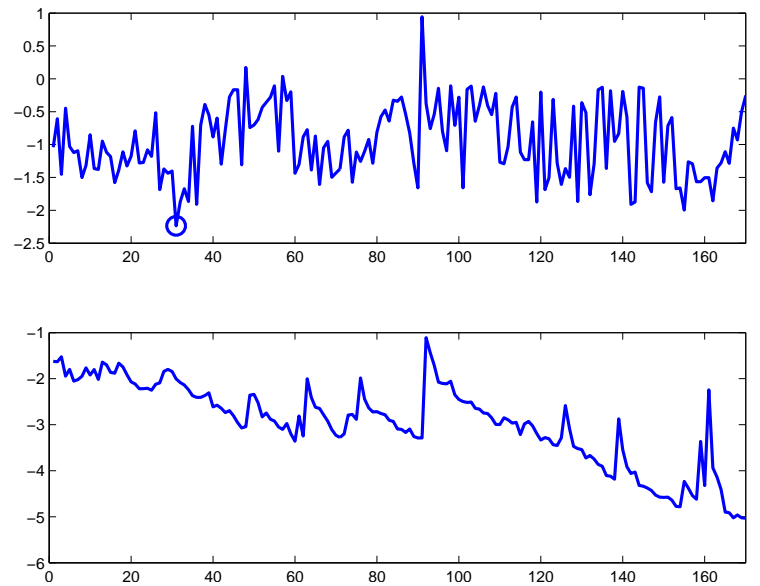

Figure 5. Defect No. \#4. Top: $\log$ of the current similarity function value. Minimizer is marked. Bottom: $\log$ of the maximal expected improvement. Both diagrams are in the function of the iteration number.

emulator, a surrogate model. This kind of surrogate modeling of electromagnetic phenomena has been a popular method for years and now seems to be having its "second honeymoon" [9]. Several methods exist for the solution of electromagnetic optimization problems. The main novelty of this paper is the introduction of such an existing (and widely used in other domains) optimization method in the domain of the ECT inverse problems.

In the developed inversion algorithm, the forward solver and the optimization method fits to each other in a natural way. The applied volume integral method provides no gradient information of the similarity function - but the optimization part does not need it at all, since the EI is a derivative-less algorithm. The EI algorithm helps us to choose the next "best" evaluation point among a discrete set of points on the parameter space. The method of variating the number of the defect cells is just a discrete variational problem, which can be easily transformed to an EI optimization task.

A pitfall of the EI algorithm is the very well-known problem of all iterative loops: setting the stopping criterion correctly is not easy and no general solution has been found yet. We have seen that the maximal expected improvement can give us a good guess whether it is worth to continue the iterations - but it was also shown that a fixed small number as the stopping limit of the maximal EI in one case can make the iteration stop before finding the minimizer while in other case it causes several "unnecessary" iterations after finding the solution - even if the configurations in the two cases are very similar.

In this paper, only the brief introduction of the stochastic tools and the first results were presented - the research is far from being finished. In the authors' opinion, the use of surrogate models in ECT inversion is a hopeful idea.

\section{Acknwoledgements}

The eddy current part of the CIVA platform has been applied, courtesy of CEA LIST, Saclay.

\section{REFERENCES}

[1] J. Bowler and S. Jenkins, "Eddy-current probe impedance due to a volumetric flaw," Journal of Applied Physics, vol. 70, no. 3, pp. 1107-1114, February 1991.
[2] J. Chiles and P. Delfiner, Geostatistics, Modeling Spatial Uncertainty. Wiley, 1999

[3] J. Sacks, W. Welch, T. Mitchell, and H. Wynn, "Design and analysis of computer experiments," Statist. Science, vol. 4, pp. 409-435, 1989.

[4] J. Villemonteix, E. Vazquez, and E. Walter, "An informational approach to the global optimization of expensive-to-evaluate functions," Journal of Global Optimization, 2007, accepted.

[5] D. Jones, "A taxonomy of global optimization methods based on response surfaces," Journal of Global Optimization, vol. 21, pp. 345383, 2001

[6] M. Locatelli, "Bayesian algorithms for one dimensional global optimization," Journal of Global Optimization, vol. 10, pp. 57-76, 1997.

[7] E. Vazquez and J. Bect, "On the convergence of the expected improvement algorithm," arXiv:0712.3744v1, 2007.

[8] CIVA: simulation software for Non Destructive Testing, http://wwwciva.cea.fr.

[9] J. K. Sykulski, "New trends in optimization in electromagnetics," Przeglad Elektrotechniczny, vol. 83, no. 6, pp. 13-18, 2007, invited paper from ISTET'07 Conference. 